\title{
Manifestations of the electron orbital moment in high-frequency properties of ferroelectrics with $3 d$-ions
}

\author{
I.E.Chupis \\ B.I.Verkin Institute For Low Temperature Physics \& Engineering \\ of National Academy of Sciences of Ukraine, \\ 47 Lenin Ave., 310164 Kharkiv, Ukraine
}

Received June 28, 1998

\begin{abstract}
A phenomenological theory of high-frequency properties of ferroelectric and ferroelectric-ferromagnet with the $3 d$-ions has been elaborated based on the separate accounting for spin and orbital electron moments, electron and ion contributions to electric polarization. In the ferroelectric state, the existence of the electron orbital moment leads to the breaks in the temperature dependencies of the transverse components of dielectric susceptibility at the ferroelectric transition temperature. The values of these breaks are proportional to the square of the electron part of spontaneous polarization and the parameter of freezing of the orbital moment. Similar breaks and the decrease of the phonon frequencies in the ferroelectric state should occur in the modes which are not soft. Besides, the effect of induction of high-frequency orbital moment by electron part of electric polarization has been predicted as well. This effect would lead to the break in the temperature dependence of paramagnetic susceptibility at ferroelectric transition temperature. In the ferroelectromagnetic state, the electron orbital moment also manifests itself in $\lambda$ increase of magnetoelectric gyration ( $\lambda$ is the constant of spin-orbital interaction).
\end{abstract}

Key words: electron, orbital, moment, ferroelectric, susceptibility

PACS: $78.20 . L S$

\section{Introduction}

In the $3 \mathrm{~d}$-dielectric, electrons of the $3 \mathrm{~d}$-shell have a weaker connection with the core than the rest electrons. This permits us to separately consider the contributions to the electric polarization from the $3 \mathrm{~d}$-electrons and from the rest part of the ion. The contribution of the $3 \mathrm{~d}$-electrons to electric polarization is essential[1]. The electron orbital moment of the $3 \mathrm{~d}$-ions in crystals is strongly frozen and its contribution to the total magnetic moment is small. A nonzero value of the orbital moment is due to a small spin-orbital interaction and makes up some percent out of the total 
moment. A small spin-orbital interaction in the 3d-ions permits to consider spin and orbital moments of the electron separately [2,3]. Thus there is a possibility to analyze the role of the electron orbital moment in forming high-frequency properties of ferroelectrics (FE) with the 3d-ions.

In the present paper a phenomenological theory of high-frequency properties of FE and ferroelectric-ferromagnet with the 3d-ions has been elaborated. The expressions obtained for the susceptibilities and spectrum contain characteristics of the electron spectrum, i.e. a spin-orbital interaction constant $\lambda$ and the parameter of the hardness of freezing of the orbital moment. The existence of the electron orbital moment leads to the breaks in the temperature dependencies of the components of dielectric susceptibility in the directions perpendicular to the spontaneous polarization at FE transition temperature. The values of these breaks are proportional to the square of the electron polarization and the parameter of freezing of the orbital moment. Besides, the breaks and the decrease of the frequencies in FE state should be in the modes which are not soft. The induction of high-frequency orbital moment by the electron part of electric polarization has been predicted as well. In the ferroelectromagnetic state, the electron orbital moment displays itself in $\lambda$-increase of magnetoelectric (ME) gyration[4].

\section{Hamiltonian and excitations}

Within the phenomenological theory a ferroelectric-ferromagnet can be described by densities of spin $\mathbf{S}(\mathbf{r})$, electron orbital $\mathbf{L}(\mathbf{r})$, ion electric $\mathbf{P}_{\mathbf{i}}(\mathbf{r})$, and electron electric $\mathbf{P}_{\mathbf{e}}(\mathbf{r})$ dipole moments as well as by densities of ion $\boldsymbol{\Pi}_{\mathbf{i}}(\mathbf{r})$ and electron $\boldsymbol{\Pi}_{\mathbf{e}}(\mathbf{r})$ momenta. To avoid too cumbersome expressions, an ion orbital moment which is much smaller than that of an electron is not taken into account here. The operators with the following nonzero commutation relations [5] correspond to the mentioned variables, i.e.

$$
\begin{aligned}
{\left[\hat{S}_{k}(\mathbf{r}), \hat{S}_{m}\left(\mathbf{r}^{\prime}\right)\right]=} & \mathrm{i} \epsilon_{k m n} \hat{S}_{n}(\mathbf{r}) \delta\left(\mathbf{r}-\mathbf{r}^{\prime}\right) \\
{\left[\hat{L}_{k}(\mathbf{r}), \hat{M}_{m}\left(\mathbf{r}^{\prime}\right)\right]=} & \mathrm{i} \epsilon_{k m n} \delta\left(\mathbf{r}-\mathbf{r}^{\prime}\right) \hat{M}_{n}(\mathbf{r}), \quad \hat{\mathbf{M}}=\hat{\mathbf{L}}, \hat{\mathbf{P}}_{\mathbf{e}}, \hat{\Pi}_{\mathbf{e}} \\
{\left[\hat{P}_{s k}(\mathbf{r}), \hat{\Pi}_{s m}\left(\mathbf{r}^{\prime}\right)\right]=} & \mathrm{i} q_{s} \Delta_{k m} \delta\left(\mathbf{r}-\mathbf{r}^{\prime}\right), \quad s=i, e \\
& q_{e}=e \hbar v_{0}^{-1}, \quad q_{i}=e_{i} \hbar v_{0}^{-1}
\end{aligned}
$$

Here indices $\mathrm{k}, \mathrm{m}, \mathrm{n}$ number the vector projections, $\Delta_{k m}$ is the Kroneker symbol; $v_{0}$ is the volume of an elementary cell; $e$ and $e_{i}$ are electron and ion charges, respectively.

Hamiltonian of a ferroelectric-ferromagnet crystal which is taken uniaxial, to be specific,(though it is not of fundamental importance) in external electric e and magnetic $\mathbf{h}$ fields is written in the form:

$$
\begin{aligned}
\hat{H} & =\int\left[\hat{H}_{m}(\mathbf{r})+\hat{H}_{e}(\mathbf{r})+\hat{H}_{e m}(\mathbf{r})\right] \mathrm{d} \mathbf{r}, \\
\hat{H}_{m} & =-\frac{b}{2} \hat{S}_{z}^{2}+\frac{\alpha}{2} \hat{\mathbf{S}}_{x_{k}}^{2}-\mu_{0} \mathbf{h}(\hat{\mathbf{L}}+2 \hat{\mathbf{S}})
\end{aligned}
$$




$$
\begin{aligned}
& +\frac{r_{\|}}{2} \hat{L}_{z}^{2}+\frac{r_{\perp}}{2}\left(\hat{L}_{x}^{2}+\hat{L}_{y}^{2}\right)+\frac{\lambda_{1}}{2} \hat{\mathbf{L}}_{x_{k}}^{2}+\lambda \hat{\mathbf{L}} \hat{\mathbf{S}} \\
\hat{H}_{e}= & -\frac{k_{1}}{2} \hat{P}_{i z}^{2}+\frac{k_{2}}{2}\left(\hat{P}_{i x}^{2}+\hat{P}_{i y}^{2}\right)+\frac{\delta}{4} \hat{P}_{i z}^{4}+\frac{\zeta}{2} \hat{\mathbf{P}}_{i_{x_{k}}}^{2} \\
& -\frac{q_{1}}{2} \hat{P}_{e z}^{2}+\frac{q_{2}}{2}\left(\hat{P}_{e x}^{2}+\hat{P}_{e y}^{2}\right)+\frac{\xi}{2} \hat{\mathbf{P}}_{e_{x_{k}}}^{2}+v_{1} \hat{P}_{e z} \hat{P}_{i z} \\
& +v_{2}\left(\hat{P}_{e x} \hat{P}_{i x}+\hat{P}_{e y} \hat{P}_{i y}\right)+\frac{1}{2 f_{e}} \hat{\boldsymbol{\Pi}}_{e}^{2}+\frac{1}{2 f_{i}} \hat{\boldsymbol{\Pi}}_{i}^{2}-\mathbf{e}\left(\hat{\mathbf{P}}_{i}+\hat{\mathbf{P}}_{e}\right), \\
\hat{H}_{e m}= & \sigma\left(\hat{\mathbf{P}}_{i}+\hat{\mathbf{P}}_{e}\right) \cdot\left[\hat{\mathbf{\Pi}}_{e} \times(\hat{\mathbf{L}}+2 \hat{\mathbf{S}})\right] .
\end{aligned}
$$

In Hamiltonian $\hat{H}_{m}$ the first term is the energy of spin-dipole interaction; the terms with coefficients $r_{\|}$and $r_{\perp}$ derived from Coulomb interactions and $r_{\|}, r_{\perp}$ are called the parameters of hardness of freezing of the orbital moment $[3] ; \lambda$ is the constant of spin-orbital interaction; $\mu_{0}$ is the Bohr magneton. The terms with coefficients $v_{1}$ and $v_{2}$ in electrodipole energy operator $\hat{H}_{e}$ describe the interaction of the electric dipole moment of the electrons of the $3 \mathrm{~d}$-shell $\mathbf{P}_{e}$ with the dipole moment of an ion core $\mathbf{P}_{i}$. The terms containing operators of momenta $\hat{\boldsymbol{\Pi}}_{e}, \hat{\boldsymbol{\Pi}}_{i}$ are the density operators of electron and ion kinetic energies, and the constants $f_{e}, f_{i}$ are proportional to electron and ion masses, respectively. ME energy (the last term $\hat{H}_{e m}$ in (2)) is of dynamic nature. This is the energy of electric polarization in an effective electric field $\mathbf{E}_{e f}$ formed by the electron moving with the velocity $\mathbf{v}$ in an internal magnetic field with induction $\mathbf{B}, \mathbf{E}_{e f}=-c^{-1} \mathbf{v} \times \mathbf{B}, c$ is the light velocity. In our case $\mathbf{v}=\boldsymbol{\Pi}_{e} v_{0} m_{e}^{-1}$ ( $m_{e}$ is the effective electron mass), $\mathbf{B}=4 \pi \mu_{0}(\mathbf{L}+2 \mathbf{S})$. Therefore, for a constant $\sigma$ in (2) one can obtain

$$
\sigma=4 \pi \mu_{0} v_{0}\left(m_{e} c\right)^{-1}
$$

The indicated ME energy is the scalar, i.e. it is present in the energy of a crystal of any symmetry. The potential ME energy (see, for instance, [2]) for the ground state considered below leads merely to the inessential renormalization of the constants in (2) and here this energy is omitted.

According to modern views a FE transition is a special case of structural phase transition. The displacement of the ionic core from the equilibrium position is accompanied by deformation of the $3 \mathrm{~d}$-shell, i.e. by electron polarization. It is assumed here that magnetic ordering arises in a spin subsystem, and due to spin-orbital interaction it magnetizes the orbital moments thus creating an average orbital moment $\mathbf{L}_{0}$ which differs from zero. All equilibrium moments $\left(\mathbf{S}_{0}, \mathbf{L}_{0}, \mathbf{P}_{o i}, \mathbf{P}_{o e}\right)$ are considered to be directed along the easy axis $Z$ of a crystal. Their magnitudes determined by minimization of homogeneous energy which corresponds to Hamiltonian (2) are as follows:

$$
L_{0}=-\lambda r_{\|}^{-1} S_{0}, \quad P_{e o}=v_{1} q_{1}^{-1} P_{i o}, \quad P_{i o}^{2}=\delta^{-1}\left(k_{1}-v_{1}^{2} q_{1}^{-1}\right),
$$

where $v_{1}^{2} \leqslant k_{1} q_{1} ; \delta, r_{\|}, r_{\perp}, b, k_{1}, k_{2}, q_{1}, q_{2}$ are positive.

To find a linear response of a ferroelectric-ferromagnet to an external electromagnetic field $(\mathbf{e}, \mathbf{h})$ a quantum-mechanical equation of motion for an operator $\partial \hat{a} / \partial t=\mathrm{i} \hbar^{-1}[\hat{H}, \hat{a}]$ is used.Taking $a \sim \exp \left[\mathrm{i}\left(\mathbf{k r}-\epsilon t \hbar^{-1}\right](\mathbf{k}\right.$ is the wave vector $)$ 
and using (1) and (2), in a linear approximation over small deviations of moments from equilibrium values, one can obtain the following equations

$$
\begin{aligned}
\left(L_{0} A_{o}-\epsilon\right) l^{+}+c_{2} P_{o e} p_{e}^{+}+v_{2} P_{o e} p_{i}^{+}+\lambda L_{0} s^{+}-\mathrm{i} \sigma_{0} \pi_{e}^{+} & =P_{o e} e^{+}+\mu_{0} L_{0} h^{+}, \\
(A-\epsilon) s^{+}+\lambda S_{0} l^{+}+2 \mathrm{i} \sigma S_{0} P_{0} \pi_{e}^{+} & =2 \mu_{0} S_{0} h^{+} \\
\bar{A}_{0} P_{o e} l^{+}+\bar{\lambda} P_{o e} s^{+}+\left(\epsilon-\epsilon_{\sigma}\right) p_{e}^{+}-\epsilon_{\sigma} p_{i}^{+}-\mathrm{i} q_{e} f_{e}^{-1} \pi_{e}^{+} & =\mu_{0} P_{o e} h^{+} \\
\left(\epsilon^{2}-\epsilon_{\perp i}^{2}\right) p_{i}^{+}-v_{2} Q_{i} p_{e}^{+}-\mathrm{i} \epsilon_{\sigma} q_{i} f_{i}^{-1} \pi_{e}^{+} & =-Q_{i} e^{+} \\
c_{2} p_{e}^{+}+v_{2} p_{i}^{+}+\mathrm{i}\left(\epsilon_{\sigma}-\epsilon\right) q_{e}^{-1} \pi_{e}^{+} & =e^{+} \\
\left(\epsilon^{2}-\epsilon_{z i}^{2}\right) p_{z i}-v_{1} Q_{i} p_{z e} & =-Q_{i} e_{z} \\
\left(\epsilon^{2}-\epsilon_{z e}^{2}\right) p_{z e}-v_{1} Q_{e} p_{z i} & =-Q_{e} e_{z} \\
\pi_{n i} & =-\mathrm{i} \epsilon f_{i} q_{i}^{-1} p_{n i}, n=x, y, z
\end{aligned}
$$

Here

$$
\begin{aligned}
& M^{+}=M_{x}+\mathrm{i} M_{y}, \quad A_{0}=r_{\perp}+\lambda_{1} k^{2}, \quad \bar{A}_{0}=A_{0}+\sigma q_{e}\left(1+q_{1} v_{1}^{-1}\right), \\
& A=S_{0}\left(b+\lambda^{2} r_{\|}^{-1}+\alpha k^{2}\right), \quad C_{n}=q_{n}+\xi k^{2}, \quad n=1,2, \\
& B_{1}=-k_{1}+3 \delta P_{0 i}^{2}+\zeta k^{2}, \quad B_{2}=k_{2}+\zeta k^{2}, \quad \sigma_{0}=\sigma\left(L_{0} P_{i 0}-2 S_{0} P_{e o}\right), \\
& \epsilon_{\sigma}=\sigma q_{e} I_{0}, \quad I_{0}=L_{0}+2 S_{0}, \quad P_{0}=P_{o i}+P_{o e}, \quad \bar{\lambda}=\lambda+l 2 \sigma q_{e}\left(1+q_{1} v_{1}^{-1}\right), \\
& \epsilon_{z i}^{2}=Q_{i} B_{1}, \quad \epsilon_{i \perp}^{2}=Q_{i} B_{2}, \quad \epsilon_{z e}^{2}=Q_{e} C_{1}, \quad Q_{i}=q_{i}^{2} f_{i}^{-1}, \quad Q_{e}=q_{e}^{2} f_{e}^{-1} .
\end{aligned}
$$

Equations for $M^{-}=M_{x}-\mathrm{i} M_{y}$ are obtained from equations (5) by the complex conjugation and the change of $\epsilon$ by $(-\epsilon)$.

Two last equations in (5) describe Z-components of excitations of the electron and the ion polarizations which in a linear approximation are not connected with the rest of the variables. Spectral branches $\epsilon_{z(1,2)}$ where

$$
\epsilon_{z(1,2)}^{2}=\frac{1}{2}\left[\epsilon_{z i}^{2}+\epsilon_{z e}^{2} \mp \sqrt{\left(\epsilon_{z e}^{2}-\epsilon_{z i}^{2}\right)^{2}+4 Q_{i} Q_{e} v_{1}^{2}}\right]
$$

correspond to these excitations. Electron dielectric susceptibility $\chi_{z z}^{e}=\partial P_{z e} / \partial e_{z}$ and ionic dielectric susceptibility $\chi_{z z}^{i}=\partial P_{z i} / \partial e_{z}$ are as follows:

$$
\begin{aligned}
& \chi_{z z}^{e}=-Q_{e}\left(\epsilon^{2}-\epsilon_{z i}^{2}+v_{1} Q_{i}\right)\left(\epsilon^{2}-\epsilon_{z 1}^{2}\right)^{-1}\left(\epsilon^{2}-\epsilon_{z 2}^{2}\right)^{-1}, \\
& \chi_{z z}^{i}=-Q_{i}\left(\epsilon^{2}-\epsilon_{z e}^{2}+v_{1} Q_{e}\right)\left(\epsilon^{2}-\epsilon_{z 1}^{2}\right)^{-1}\left(\epsilon^{2}-\epsilon_{z 2}^{2}\right)^{-1} .
\end{aligned}
$$

As the constants $f_{e}$ and $f_{i}$ are proportional to electron $\left(m_{e}\right)$ and ion $\left(m_{i}\right)$ masses, respectively, then the ratio of frequencies $\left(\omega=\hbar^{-1} \epsilon\right)$ of electron and ion excitations (7) is of the order of $\omega_{e} / \omega_{i} \sim\left(m_{i} / m_{e}\right)^{1 / 2} \gg 1$. The lower branch of the spectrum is practically a branch of ion excitations, and the upper one corresponds to electron excitations. Using equations (4), (6) and (7) it is easy to see that at FE phase transition $\left(P_{0}=0\right)$, the activation energy of the lower branch $\epsilon_{z 1}$ turns to zero. The "twinning law" is fulfilled for the total static FE susceptibility $\chi_{z z}=\chi_{z z}^{i}+\chi_{z z}^{e}$. If $v_{1}=0$ in expressions (8), then for static susceptibilities from (7) and (8) one finds 
$\chi_{z z}^{e}(0)=C_{1}^{-1}, \chi_{z z}^{i}(0)=B_{1}^{-1}$. In the frequency range $\omega_{e} \gg \omega$, the electron susceptibility changes slightly (as compared to $\chi_{z z}^{e}(0)$ ) while the ion susceptibility is of resonance behaviour near $\omega_{z 1}$. At $v_{1} \neq 0$ both ion and electron susceptibilities are of resonance behaviour near $\omega_{z 1}$. At high frequencies $\omega \gg \omega_{i}$, the electron susceptibility is $\left(\omega_{e} / \omega_{i}\right)^{2}$ times larger than the ion susceptibility. At these frequencies, $\chi_{z z}^{e}$ being far from resonance has got the same order of magnitude as in a static case.

Excitations of the components of moments perpendicular to their equilibrium direction, namely, to the axis Z, are connected with each other. From equations (5) one can obtain expressions for generalized susceptibility,i.e. electric $\chi_{n k}=\partial P_{n} / \partial e_{k}$, magnetic $x_{n k}=\mu_{0} \partial I_{n} / \partial h_{k}$, magnetoelectric $\alpha_{n k}^{m e}=\mu_{0} \partial I_{n} / \partial e_{k}, \alpha_{n k}^{e m}=\partial P_{n} / \partial h_{k}$, where $\mathbf{P}=\mathbf{P}_{i}+\mathbf{P}_{e}, \mathbf{I}=\mathbf{L}+2 \mathbf{S}$. In the absence of damping $\alpha_{n k}^{e m}=\left(\alpha_{k n}^{m e}\right)^{\star}$.

The total expressions for susceptibilities are too cumbersome and it is convenient to analyze them for different states of the system.

\section{Ferroelectric state}

If a spontaneous magnetic moment isn't present, $\left(S_{0}=L_{0}=0\right)$, and $P_{0} \neq 0$, then there are four branches of excitations, namely, $\epsilon_{z 1}, \epsilon_{z 2}(7)$ and optical ion and electron excitations $\mathbf{p}_{\perp}$ in the basic plane with the energies $\epsilon_{o i}, \epsilon_{o e}$ respectively. The spectrum is degenerated, i.e.the excitations $p_{x}$ and $p_{y}$ have the same energy and are of oscillation character. The energies $\epsilon_{o i}$ and $\epsilon_{o e}$ determined from equations (5) are as follows:

$$
\epsilon_{o(i, e)}^{2}=\frac{1}{2}\left[\epsilon_{i \perp}^{2}+\epsilon_{e \perp}^{2} \mp \sqrt{\left(\epsilon_{e \perp}^{2}-\epsilon_{i \perp}^{2}\right)^{2}+4 Q_{i} \bar{Q}_{e} v_{2}^{2}}\right] .
$$

Here $\epsilon_{e \perp}^{2}=C_{2} \bar{Q}_{e}$, where $\bar{Q}_{e}=Q_{e}-\bar{A}_{0} P_{o e}^{2}$.

As $\epsilon_{e \perp} \gg \epsilon_{i \perp}$ we obtain approximately

$$
\epsilon_{o i}^{2} \approx Q_{i}\left(C_{1}-v_{2}^{2} C_{2}^{-1}\right), \quad \epsilon_{o e}^{2} \approx \epsilon_{e \perp}^{2} \approx C_{2}\left(Q_{e}-\bar{A}_{0} P_{o e}^{2}\right) .
$$

In $\mathrm{FE}$ state in the energy of transverse electron mode $\epsilon_{e \perp}(10)$ the term appears which is proportional to the square of the electron part of polarization and the parameter of freezing of orbital moment since $\bar{A}_{0}(k=0)=r_{\perp} \geqslant 0$. This means the decrease and the break in the temperature dependence of transverse electron frequency which is not soft at FE transition.

Transverse components of ion $\chi_{n k}^{i}$ and electron $\chi_{n k}^{e}$ dielectric susceptibilities are as follows:

$$
\begin{aligned}
\chi_{x x}^{i}=\chi_{y y}^{i} & =-Q_{i} D\left(\epsilon^{2}-\epsilon_{e \perp}^{2}+v_{2} \bar{Q}_{e}\right), \\
D & =\left(\epsilon^{2}-\epsilon_{o i}^{2}\right)^{-1}\left(\epsilon^{2}-\epsilon_{o e}^{2}\right)^{-1}, \\
\chi_{x x}^{e}=\chi_{y y}^{e} & =-\bar{Q}_{e} D\left(\epsilon^{2}-\epsilon_{i \perp}^{2}+v_{2} Q_{i}\right) .
\end{aligned}
$$

One sees from (11) that transverse components of dielectric susceptibilities as well as the frequency have got breaks at FE transition temperature. The break magnitude will have the largest value at high frequencies $\omega \sim \omega_{e}$,

$$
\Delta \chi_{x x}^{e}=\chi_{-}^{e}-\chi_{+}^{e}=\bar{A}_{0} P_{0 e}^{2} \epsilon^{2}\left(\epsilon^{2}-\epsilon_{e \perp}^{2}\right)^{-2},
$$


where $\Delta \chi$ is the difference between the susceptibility $\left(\chi_{+}^{e}\right)$ above FE transition temperature $T_{e}$ extrapolated to $T \leqslant T_{e}$ and the susceptibility $\chi_{-}^{e}$ below $T_{e}$. According to the order of magnitude $\bar{A}_{0} \sim \epsilon_{e}$, thus $\Delta \chi \sim P_{0 e}^{2} \epsilon_{e}^{-1}$. An experimental measurement of the mentioned breaks could help to evaluate the parameters $P_{0 e}$ and $r_{\perp}$.

As it follows from the first equation in (5) excitations of electric polarization $\mathbf{p}_{\perp}$ at $P_{0} \neq 0$ are accompanied by excitations of an orbital moment $\mathbf{l}_{\perp}$, i.e. high-frequency linear ME effect takes place [4]. This effect is characterized by a nondiagonal component of ME susceptibility

$$
\left.\alpha_{x y}^{e m}=-\mathrm{i} \epsilon \mu_{0} P_{0 e}\left(\epsilon^{2}-\epsilon_{i \perp}^{2}+v_{2} Q_{i}\right)\left(\epsilon^{2}-\bar{\epsilon}_{0 i}^{2}\right)^{-1}\right)\left(\epsilon^{2}-\bar{\epsilon}_{o e}^{2}\right)^{-1} .
$$

Besides, the orbital magnetic susceptibility which is proportional to the square of electron spontaneous polarization is induced:

$$
x_{x x}^{0}=x_{y y}^{0}=\mu_{0}^{2} P_{0 e}^{2}\left[C_{2}\left(\epsilon^{2}-\epsilon_{i \perp}^{2}\right)+v_{2}^{2} Q_{i}\right]\left(\epsilon^{2}-\bar{\epsilon}_{o i}^{2}\right)^{-1}\left(\epsilon^{2}-\bar{\epsilon}_{o e}^{2}\right)^{-1} .
$$

The effect of induction of the orbital magnetic susceptibility in a FE state should be accompanied by a break in temperature dependence of paramagnetic susceptibility at $T=T_{e}$. According to (14), the break magnitude is proportional to the square of electron polarization.

The value of ME susceptibility (13) increases with the increasing frequency (i.e. $\epsilon$ ) and it is a resonance behaviour at ion $\left(\bar{\omega}_{o i}\right)$ and electron $\left(\bar{\omega}_{o e}\right)$ frequencies. The largest value $\alpha_{x y}$ takes at electron frequencies $\omega \sim \omega_{e}$ [4]. In this case far from resonance $\alpha_{x y} \sim \mu_{0} P_{0 e} \epsilon_{e}^{-1}$. For values $P_{0 e} \sim 10 \mu \mathrm{C} \mathrm{cm}^{-2}$ the magnitude $\alpha_{x y} \sim 10^{-4}-10^{-3}$. Orbital magnetic susceptibility induced by spontaneous electric polarization is much smaller.

\section{Ferroelectric-ferromagnetic state}

In the ferroelectric-ferromagnetic state when $S_{0} \neq 0, L_{0} \neq 0, P_{0} \neq 0$, the optical phonon excitations $\mathbf{p}_{\perp}$ are not degenerated and are of circle precession character around the direction of a spontaneous magnetic moment $I_{o z}=L_{o z}+2 S_{o z}$. The energies left $\left(\epsilon_{-}\right)$and right $\left(\epsilon_{+}\right)$precessions are different. As the values of $\epsilon_{e \perp} \gg$ $\epsilon_{i \perp} \gg \epsilon_{\sigma}$ from (5) we obtain in a linear approximation over $\epsilon_{\sigma}$

$$
\begin{aligned}
\epsilon_{i} & \approx \epsilon_{o i} \pm \epsilon_{\sigma} q_{i} v_{2}\left(2 f_{i}\right)^{-1} \epsilon_{o e}^{-2}\left(q_{i}+q_{e}-2 q_{i} v_{2} C_{2}^{-1}\right), \\
\epsilon_{e} & \approx \epsilon_{o e} \pm \epsilon_{\sigma} .
\end{aligned}
$$

As seen from (15),splitting of lines of the ion phonon spectrum in the internal magnetic field $\sim I_{o}$ is $\left(\epsilon_{e} / \epsilon_{i}\right)$ times smaller than the electron one. The latter is also rather small, $\sim \epsilon_{\sigma} / \epsilon_{e}$.

Magnetic moment $I_{o}$ induces a nondiagonal component of dielectric susceptibility $\chi_{x y}$. In the same linear approximation over $\epsilon_{\sigma}$ one finds

$$
\chi_{x y}^{i}=\chi_{y x}^{i \star}=-\mathrm{i} \epsilon_{\sigma} \epsilon Q_{i} D^{2}\left\{q_{e}\left(\epsilon^{2}-\epsilon_{i \perp}^{2}\right)\left(\epsilon^{2}-\epsilon_{e \perp}^{2}+2 q_{i} q_{e} v_{2} f_{e}^{-1}\right)\right.
$$




$$
\begin{array}{cl} 
& \left.+3 q_{i} q_{e} v_{2}^{2} Q_{i} \bar{Q}_{e}+v_{2} Q_{i}\left(q_{i}+q_{e}\right)\left(\epsilon^{2}-\epsilon_{e \perp}^{2}\right)\right\}, \\
\chi_{x y}^{e}=\chi_{y x}^{e \star}= & -\mathrm{i} \epsilon_{\sigma} \epsilon D^{2}\left\{\left(\epsilon^{2}-\epsilon_{i \perp}^{2}\right)\left[Q_{i}\left(\epsilon^{2}-\epsilon_{e \perp}^{2}\right)+2 \bar{Q}_{e}\left(\epsilon^{2}-\epsilon_{i \perp}^{2}\right)\right]\right. \\
& \left.\left.+q_{i} f_{i}^{-1} v_{2} \bar{Q}_{e}\left(3 q_{i}+q_{e}\right)\right]+q_{e} q_{i}^{-1} v_{2}^{2} \bar{Q}_{e} Q_{i}^{2}\right\} .
\end{array}
$$

It follows from (16) that spin and orbital moments induce electric gyrotropy, $\chi_{x y} \sim$ $\left(L_{o}+2 S_{o}\right)$ which increases with the increasing frequency. The estimations of expressions (16) far from resonance give $\chi_{x y}^{i} \sim \chi_{x y}^{e} \sim \epsilon_{\sigma} \epsilon_{s} / \epsilon_{e}^{2}\left(\epsilon \sim \epsilon_{s}\right), \chi_{x y}^{i} \sim \chi_{x y}^{e} \sim$ $\epsilon_{\sigma} \epsilon_{i} / \epsilon_{e}^{2}\left(\epsilon \sim \epsilon_{i}\right)$. At frequencies $\omega \sim \omega_{e} \sim 10^{14}-10^{15} \mathrm{rad} \mathrm{s}^{-1}$ the electron contribution to dielectric susceptibility is considerably larger than the ion contribution, and the electric gyrotropy is of the order of magnitude $\chi_{x y}^{e} \sim \epsilon_{\sigma} / \epsilon_{e}$. The magnitude $\epsilon_{\sigma}=8 \pi \mu_{o}^{2} I_{o} \sim 10 \epsilon_{s}$, i.e. the largest value is $\chi_{x y}^{e} \sim 10^{-3}$.

High-frequency ME susceptibility of uniaxial ferroelectric-ferromagnet has got three components which differ from zero, i.e. $\alpha_{x y}$ and $\alpha_{x x}=\alpha_{y y}$. A nondiagonal component $\alpha_{x y}$ is approximately determined by expressions (13) at frequencies which are larger than the spin ones.

A new component $\alpha_{x x}=\alpha_{y y}$ which differs from zero appears only in a ferroelectric-ferromagnetic state ( or in external electric and magnetic fields[4]), $\alpha_{x x} \sim$ $P_{0} S_{0}$. At the frequencies $\omega \sim \omega_{e}$ where ME susceptibility is the largest we obtain approximate expressions

$$
\begin{aligned}
\alpha_{x x}^{e m}=\alpha_{y y}^{e m} & \simeq \mu_{0} P_{0 e}\left(\epsilon_{0}+2 \bar{\lambda} S_{0}\right)\left(\epsilon^{2}-\epsilon_{0 e}^{2}\right)^{-1} \\
\alpha_{x y}^{e m} & \simeq-i \mu_{0} \epsilon P_{0 e}\left(\epsilon^{2}-\epsilon_{0 e}^{2}\right)^{-1} .
\end{aligned}
$$

In [4] where FE was considered in a constant external magnetic field, the expression obtained for $\alpha_{x x}$ differs from (17). This difference is in the following. There is an energy of an external magnetic field $\mu_{0} H_{0}$ instead of the energy of orbital excitations $\left(\epsilon_{0}+2 \bar{\lambda} S_{0}\right)$. For maximum fields $H_{0} \sim 10^{5}$ Oe reached at present, the energy $\mu_{0} H_{0} \sim 10^{\circ} \mathrm{K}$ whereas the value of the energy of orbital excitations $\epsilon_{0} \sim \lambda S_{0}$ for the $3 \mathrm{~d}$-compounds is considerably larger, of the order of $100 \mathrm{~K}$.

As shown in [4], the presence of ME susceptibility creates a possibility of a new optical effect - ME gyration - which consists in the rotation of a plane of polarization of the reflected and of the transmitted light by an angle which is proportional to the product of the first degrees of spontaneous electric polarization and magnetic field, i.e. to the value $P_{0 e} H_{0}$. Therefore, in a ferroelectric-ferromagnet where the value $\alpha_{x x}$ is considerably larger than in $\mathrm{FE}$, the effect of $\mathrm{ME}$ gyration is considerably strengthened by spin-orbital interaction. In other words, in ferroelectric-ferromagnet, an orbital moment manifests itself by $\lambda$-strengthening of ME gyration.

\section{Conclusions and summary}

Therefore, a simultaneous consideration of the spin, of the orbital moment, of the electron polarization and of the ion polarization of the $3 \mathrm{~d}$-compounds has provided a possibility to analyze possible manifestations of electron shell polarization and 
orbital degrees of freedom of the 3d-electrons in a spectrum and high-frequency susceptibility.

The orbital moment manifests itself in the breaks in the temperature dependencies of a nonsoft phonon mode and transverse dielectric susceptibility at FE transition temperature and in $\lambda$-increase of ME-gyration. Besides, ME effect of induction of high-frequency orbital susceptibility by the electron part of electric polarization has been also predicted. This effect should lead to the break in the temperature dependence of paramagnetic susceptibility at FE transition temperature.

In a series of publications [6-8] the observation was reported of a "magnetopolarization gyration" in ferroelectrics manifested in the rotation of the polarization plane of the light propagating along the magnetic field and the optical axis of the crystal through an angle $\phi$ proportional to the product of the first powers of electric polarization and magnetic field. By contrast to Faraday's effect, the double (forward and backward) passage of light has led to a compensation of the above-mentioned "magnetopolarization" effect. On the first sight this effect seems similar to ME gyration [4] but the estimates of ME gyration give a much smaller magnitude of the effect. In a ferroelectric-ferromagnet, the orbital moment increases the effect of $\mathrm{ME}$ gyration in $\lambda \sim 10^{2}$ times in comparison with the value $\phi \sim 10^{-7}$ rad in a ferroelectric [4]. However, this value $\phi \sim 10^{-5} \mathrm{rad}$ is smaller than that declared in [6-8].

As for as the values of all the predicted effects are proportional to a spontaneous electric polarization then the 3d-compounds with a proper FE transition are more useful for the experimental investigations.

This research was supported by INTAS Grant No. 94-935.

\section{References}

1. Beznosov A.B., Galuza A.I., Eremenko V.V. Valence fluctuations and atomic polarizability of iron oxides in an atomic polarizability of iron oxides. - In: "Inter. Conf. of Magnetizm", Abstract. San-Francisco, 1985, 1Pe 13, p. 67.

2. Chupis I.E., Govorun A.V. On the theory of high-frequency properties of a ferroelectromagnetic with unfrozen orbital moment. // Fiz. Nizk. Temp., 1995, vol. 21, No. 2, p. 228-234 (in Russian).

3. Rozenfeld E.V., Korolev A.V. Low temperature anisotropy of magnetization in ferromagnetic with frozen orbital moment. // Zh. Eksp. Teor. Fiz., 1995, vol. 108, No. 3(9), p. 862-877 (in Russian).

4. Chupis I.E. Magnetooptical effects in ferroelectric materials. // Fiz. Nizk. Temp., 1997, vol. 23, No. 3, p. 290-295 (in Russian).

5. Chupis I.E. Elementary excitations in ferroelectromagnetic with orbital magnetic moment. // Fiz. Tverd. Tela, 1994, vol. 36, No. 7, p. 1910-1917 (in Russian).

6. Vlokh O.G. Magnetogyration. // Ukr. Fiz. Zh., 1981, vol. 26, No. 10, p. 1623-1626 (in Russian).

7. Vlokh O.G., Zheludev I.S., Sergatyuk V.A. Electro-and magnetogyration in crystals. // Izv. Akad. Nauk SSSR, Ser. Fiz., 1984, vol. 48, No. 9, p. 1771-1776 (in Russian). 
8. Vlokh O.G., Sergatyuk V.A. Magnetopolarizational pseudogyration. // Doklady Akad. Nauk SSSR, 1986, vol. 291, No. 4, p.832-835 (in Russian).

\title{
Прояви електрон-орбітального моменту у високочастотних властивостях сегнетоелектриків 3 3d-іонами
}

\author{
І.Чупіс \\ Фізико-технічний інститут низьких температур \\ ім. Б.І.Веркіна НАН України, \\ 310164 Харків, просп. Леніна, 47 \\ Отримано 28 червня 1998 р.
}

Побудована феноменологічна теорія високочастотних властивостей сегнетоелектрика і сегнетоелектрик-феромагнетика з 3d-іонами, яка грунтується на розділеному врахуванні спінового і орбітального електронних моментів, електронних та іонних вкладів до електричної поляризації. У сегнетоелектричному стані існування електронного орбітального момента приводить до розривів поперечних компонент діелектричної сприйнятливості при температурі сегнетоелектричного переходу. Величини цих розривів пропорціональні до квадрата електронної частини спонтанної поляризації і параметра замороження орбітального момента. Аналогічні розриви і зменшення фононних частот у сегнетоелектричному стані мають спостерігатися в модах, які не є м'якими. Крім того, передбачено ефект індукції високочастотного орбітального момента на електронну частину поляризації. Цей ефект може приводити до розриву парамагнітної сприйнятливості при температурі сегнетоелектричного переходу. У фероелектромагнітному стані електронний орбітальний момент проявляє себе в $\lambda$ зростанні магнітоелектричної гірації ( $\lambda \in$ постійна спінорбітальної взаємодії).

Ключові слова: електрон, орбіталь, момент, сегнетоелектрик, сприйнятливість

PACS: $78.20 . L S$ 
